\title{
UNIQUE SOLVABILITY OF THE STRONG HAMBURGER MOMENT PROBLEM
}

\author{
OLAV NJÅSTAD and W. J. THRON
}

(Received 3 August 1983; revised 8 May 1984)

Communicated by G. Brown

\begin{abstract}
Methods from the theory of orthogonal polynomials are extended to $L$-polynomials $\sum_{n=p}^{q} a_{n} z^{n}$. By this means the authors and W. B. Jones (J. Math. Anal. Appl. 98 (1984), 528-554) solved the strong Hamburger moment problem, that is, given a double sequence $\left\{c_{n}\right\}_{-\infty}^{\infty}$, to find a distribution function $\psi(t)$, non-decreasing, with an infinite number of points of increase and bounded on $-\infty<t<\infty$, such that for all integers $n, c_{n}=\int_{-\infty}^{\infty}(-t)^{n} d \psi(t)$. In this article further methods such as analogues of the Liouville-Ostrogradski formula and of the Christoffel-Darboux formula are developed to investigate when the moment problem has a unique solution. This will be the case if and only if a sequence of nested disks associated with the sequence $\left\{c_{n}\right\}_{-\infty}^{\infty}$ has only a point as its intersection (the so called limit point case).
\end{abstract}

1980 Mathematics subject classification (Amer. Math. Soc.): 30 E 05, 33 A 65.

\section{Introduction}

By a distribution function on an interval $I$ (finite or infinite) we shall here mean a bounded, real-valued, non-decreasing function with an infinite number of points of increase. Distribution functions $\psi$ for which all the moments $c_{n}=$ $(-1)^{n} \int_{I} t^{n} d \psi(t), n=0,1,2, \ldots$, exist, are of great importance and have been extensively studied (see e.g. $[1,15]$ ). Among other things they admit Gaussian quadrature formulas, obtained by the aid of polynomials orthogonal with respect to $\psi$, which approximate the integrals $\int_{I} f(t) d \psi(t)$ for large classes of functions. The Padé approximants at $\infty$ for the series $\sum_{n=0}^{\infty} c_{n} z^{-n}$ are closely related to the same orthogonal polynomials. The integral $\int_{I} d \psi(t) /(t-z)$ defines an analytic

(c) 1986 Australian Mathematical Society $0263-6115 / 86 \$ A 2.00+0.00$ 
function $F(z)$ outside $I$, and the series $\sum_{n=0}^{\infty} c_{n} z^{-n}$ is an asymptotic expansion for $F(z)$ at $\infty$ with respect to suitable regions. The sequence of main diagonal Padé approximants has subsequences converging to functions of the form $G(z)=$ $z \int_{I} d \phi(t) /(t-z)$, where the functions $\phi$ are among the distribution functions giving rise to the same moment sequence $\left\{c_{n}: n=0,1,2, \ldots\right\}$ as $\psi$.

Among the important problems arising in the study of these connections, is the question of existence of distribution functions having a given sequence $\left\{c_{n}\right.$ : $n=0,1,2, \ldots\}$ as moments. If such a distribution function $\psi$ exists, then for example the series $\sum_{n=0}^{\infty} c_{n} z^{-n}$ may be said to have a generalized sum $F(z)=$ $\int_{I} d \psi(t) /(t-z)$, since this series is an asymptotic expansion for $F(z)$ at $\infty$. It is also of importance to know when the solution of the above moment problem is unique, i.e. when a distribution function for which all positive moments exist, is determineed by these moments.

Questions concerning two sequences $\left\{c_{n}: n=0,1,2, \ldots\right\}$ and $\left\{c_{n}: n=\right.$ $-1,-2, \ldots\}$, or equivalently double sequences $\left\{c_{n}: n=0, \pm 1, \pm 2, \ldots\right\}$, arise as natural extensions of the foregoing. Distribution functions $\psi$ having both positive and negative moments $c_{n}=(-1)^{n} \int_{I} t^{n} d \psi(t), n=0, \pm 1, \pm 2, \ldots$, admit larger classes of integrable functions than those with only positive moments. They give rise to Gaussian quadrature formulas by the aid of a theory of orthogonal $L$-polynomials (see Section 1 ), and these quadrature formulas approximate the integrals $\int_{I} f(t) d \psi(t)$ for larger classes of functions than in the classical theory. The two-point Padé approximants at $\infty$ and 0 for the series $\sum_{n=0}^{\infty} c_{n} z^{-n}$ and $-\sum_{n=1}^{\infty} c_{n} z^{n}$ are related to the same orthogonal $L$-polynomials. These series are asymptotic expansions for the function $F(z)=\int_{I} d \psi(t) /(t-z)$ at $\infty$ and 0 , respectively, with respect to suitable regions. The sequence of main diagonal Padé approximants has subsequences converging to functions of the form

$$
G(z)=z \int_{I} d \phi(t) /(t-z),
$$

where the functions $\phi$ are among the distribution functions giving rise to the same moment sequence $\left\{c_{n}: n=0, \pm 1, \pm 2, \ldots\right\}$ as $\psi$.

For similar reasons as in the classical case it is of interest to study questions of existence and uniqueness of solutions of this strong moment problem. When is a double sequence $\left\{c_{n}: n=0, \pm 1, \pm 2, \ldots\right\}$ the moment sequence of a distribution function $\psi$ ? When is a distribution function with both positive and negative moments determined by these moments?

When $I$ is the interval $[0, \infty)$, the above problem is called the Strong Stieltjes Moment Problem (SSMP), and when $I$ is the interval $(-\infty, \infty)$, the problem is called the Strong Hamburger Moment Problem (SHMP).

In [9] Jones, Thron and Waadeland began the study of strong moment problems. In that article they stated and solved the SSMP. To solve the problem the authors used continued fractions methods, in particular the theory of positive 
$T$-fractions, to show that the necessary conditions for the existence of a solution, namely

$$
H_{2 n}^{(-2 n)}>0, \quad H_{2 n+1}^{(-2 n)}>0, \quad H_{2 n}^{(-2 n+1)}>0, \quad H_{2 n+1}^{(-2 n-1)}<0,
$$

(the Hankel determinants $H_{k}^{(n)}$ are defined in Section 1) are also sufficient. In addition they obtained necessary and sufficient conditions, in terms of the positive $T$-fraction, for the solution to be unique.

Somewhat later Jones and Thron [7] pointed out that there is a sequence of orthogonal functions ( $L$-polynomials) associated with the SSMP and that the concept of Gaussian quadrature can be extended to this case. They also stated the SHMP. These ideas were then further elaborateed by Jones and the present authors in [4] to show that the positive definiteness of the bilinear form associated with the sequence $\left\{c_{n}: n=0, \pm 1, \pm 2, \ldots\right\}$ (see Section 1 ), or equivalently the determinant condition

$$
H_{2 n}^{(-2 n)}>0, \quad H_{2 n+1}^{(-2 n)}>0, \quad \text { for } n \geqslant 0,
$$

is necessary and sufficient for the existence of a solution of the SHMP.

In [5] we turned to the question to what extend the SHMP could be solved by the use of continued fractions. For those sequences $\left\{c_{n}: n=0, \pm 1, \pm 2, \ldots\right\}$ where all indices are non-singular (see Section 1) we solve the problem completely. That is, we established existence and also obtained necessary and sufficient conditions for the uniqueness of the solution. In that article we put properties of continued fractions, such as integral representation and complete convergence, in the foreground.

Properties of doubly infinite moment sequences and corresponding quadrature formulas and continued fractions expansions are also treated in [6].

In the present article we develop the machinery of orthogonal $L$-polynomials further to obtain an answer to the uniqueness question in all cases (not just in those where all indices are non-singular). Here we emphasize basic functional analytic tools and use these combined with complex variable methods. The presentation is closely related to that given in [1] for the classical situation. A treatment of the classical case where general Hilbert space methods are more systematically exploited is given in [12].

Among the central concepts discussed in this article are quasi-orthogonal and associated $L$-polynomials and analogues of the Christoffel-Darboux and Green formulas from the classical theory of orthogonal polynomials. There are however also substantial differences from the classical theory. Chief among these are the existence of singular $L$-polynomials and the non-existence of a three term recursion formula betwen successive orthogonal $L$-polynomials.

As was the case in the classical Hamburger problem the SHMP has a unique solution if and only if a certain sequence of nested discs shrinks to a limit point. There will not be a unique solution to the SSMP if the positive $T$-fraction 
determined by the moments diverges (see [9]). Hence for this sequence of moments the SHMP also must have an infinite number of solutions. In [4] we have exhibited a class of moment sequences for which the SHMP has a unique solution. Hence both alternatives are possible.

Recently other authors have also taken an interest in the SHMP. We are aware of the work of W. B. Gragg (whose approach is sketched in [8, Section 5]), Chr. Berg and A. Sri Ranga whose contributions have not as yet appeared in print. A historical survey of moment problems and related topics can be found in [8].

Other questions related to doubly infinite moment sequences have previously been considered by Cobindarajula [3], Kabe [10], Mendenhall and Lehman [11] and Thomas [16].

\section{Preliminaries}

For any pair $(p, q)$ of integers with $p \leqslant q$, let $\mathscr{R}_{p, q}$ denote the set of all functions of a complex variable $z$ of the form $R(z)=\sum_{j=p}^{q} r_{j} z^{j}, r_{p}, \ldots, r_{q}$ complex numbers. We shall write $\mathscr{R}_{2 m-1}=\mathscr{R}_{-m, m-1}, \mathscr{R}_{2 m}=\mathscr{R}_{-m, m}$, and $\mathscr{R}=\bigcup_{n=0}^{\infty} \mathscr{R}_{n}$. A function $R$ belonging to $\mathscr{R}$ is called a Laurent polynomial (or L-polynomial). The sets $\mathscr{R}_{p, q}$ and $\mathscr{R}$ form linear spaces over the complex numbers with respect to the usual operations of addition and multiplication by a scalar. A basis for each of the spaces $\mathscr{R}, \mathscr{R}_{2 m}$ and $\mathscr{R}_{2 m-1}$ is given by $\left\{1, z^{-1}, z, z^{-2}, z^{2}, \ldots\right\}$, $\left\{z^{-m}, \ldots, 1, \ldots, z^{m}\right\},\left\{z^{-m}, \ldots, 1, \ldots, z^{m-1}\right\}$, respectively.

In the following, $C=\left\{c_{n}: n=0, \pm 1, \pm 2, \ldots\right\}$ shall denote a given (double) sequence of real numbers. For each $n=0, \pm 1, \pm 2, \ldots$, the Hankel determinants $H_{k}^{(n)}$ associated with the sequence $C$ are given by

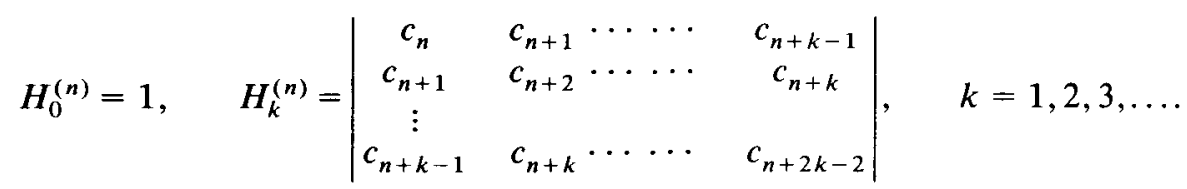

A linear functional $\gamma$ on $\mathscr{R}$ is uniquely determined by the sequence $C$ as follows:

$$
\gamma\left(\sum_{j=p}^{q} r_{j} z^{j}\right)=\sum_{j=p}^{q}(-1)^{j} r_{j} c_{j} .
$$

A solution of the SHMP for a given $C$ is a distribution function (that is, a bounded, real-valued, non-decreasing function $\psi$ with infinitely many points of increase), such that the functional $I_{\psi}$ defined by $I_{\psi}(f)=\int_{-\infty}^{\infty} f(t) d \psi(t)$ coincides with $\gamma$ on $\mathscr{R}$.

Let $\mathscr{R}_{R}$ denote the real linear space of all $L$-polynomials with real coefficients. In terms of $\gamma$ we define a fuctional $\langle$,$\rangle on \mathscr{R}_{R} \times \mathscr{R}_{R}$ by setting $\langle A, B\rangle=\gamma(A \cdot B)$ for all $A, B \in \mathscr{R}_{R}$. The SHMP has a solution if and only if the bilinear functional 
$\langle$,$\rangle is positive definite (i.e. an inner product), and a necessary and sufficient$ condition for this to be the case is that the following condition $(\mathrm{H})$ is satisfied:

$$
H_{2 m}^{(-2 m)}>0, \quad H_{2 m+1}^{(-2 m)}>0, \quad m=0,1,2, \ldots,
$$

(see [4]). From now on we shall always assume that the condition $(\mathrm{H})$ holds. By applying the Gram-Schmidt orthonormalization process to the sequence $\left\{1, z^{-1}, z, z^{-2}, z^{2}, \ldots\right\}$ we obtain an orthonormal sequence $\left\{Q_{n}(z): n=\right.$ $0,1,2, \ldots\}$ of $L$-polynomials. For each $n \geqslant 0, Q_{n} \in \mathscr{R}_{n}$ and $\left\|Q_{n}\right\|^{2}=\gamma\left(Q_{n}^{2}\right)=1$. We can write, for $m=0,1,2, \ldots$,

$$
\begin{aligned}
& Q_{2 m}(z)=\sum_{j=-m}^{m} q_{2 m, j} z^{j}, \text { where } q_{2 m, m} \neq 0, \\
& Q_{2 m+1}(z)=\sum_{j=-(m+1)}^{m} q_{2 m+1, j} z^{j}, \quad \text { where } q_{2 m+1,-(m+1)} \neq 0 .
\end{aligned}
$$

Note that $Q_{0}(z)=q_{0,0}$, where in general $q_{0,0} \neq 1$. By a standard orthogonality argument we get

$$
\begin{aligned}
& \gamma\left(z^{m} \cdot Q_{2 m}(z)\right)=\left(q_{2 m, m}\right)^{-1}, \\
& \gamma\left(z^{-(m+1)} \cdot Q_{2 m+1}(z)\right)=\left(q_{2 m+1,-(m+1)}\right)^{-1} .
\end{aligned}
$$

All zeros of $Q_{n}(z)$ are real and simple, and the number $\nu_{n}$ of zeros is either $n$ or $n-1$ (see Theorem 3.1 of [4]). The index $n$ and the $L$-polynomial $Q_{n}(z)$ are called singular if $\nu_{n}=n-1$, and non-singular if $\nu_{n}=n$. If $Q_{2 m}(z)$ is singular, then $q_{2 m,-(m-1)} \neq 0$ and

$$
Q_{2 m}(z)=\frac{q_{2 m,-(m-1)}}{q_{2 m-1,-m}} z Q_{2 m-1}(z),
$$

and if $Q_{2 m+1}(z)$ is singular, then $q_{2 m+1, m-1} \neq 0$ and

$$
Q_{2 m+1}(z)=\frac{q_{2 m+1, m-1}}{q_{2 m, m}} z^{-1} Q_{2 m}(z) \text {. }
$$

(See Corollary 3.2 and Theorem 3.3 of [4]. Note however, that the orthogonal $L$-polynomials are normalized differently here.) Two successive orthonormal $L$-polynomials $Q_{n}(z)$ and $Q_{n+1}(z)$ therefore cannot both be singular.

Let $t_{j}^{(n)}, j=1,2, \ldots, n$, be the zeros of $Q_{n}(z)$ for non-singular $n$. There exist positive constants $\lambda_{j}^{(n)}, j=1,2, \ldots, n$, such that the Gaussian quadrature formula

$$
\gamma(F)=\sum_{j=1}^{n} \lambda_{j}^{(n)} F\left(t_{j}^{(n)}\right)
$$

is valid for all $F \in \mathscr{R}_{2 m-1}$ (see Theorem 5.1 of [4]).

For complex numbers $z$ and $\tau$ we define $Q_{n}(z, \tau)$ by

$$
\begin{aligned}
& Q_{2 m}(z, \tau)=Q_{2 m}(z)-\tau z Q_{2 m-1}(z), \quad m=1,2, \ldots, \\
& Q_{2 m+1}(z, \tau)=Q_{2 m+1}(z)-\tau z^{-1} Q_{2 m}(z), \quad m=0,1,2, \ldots
\end{aligned}
$$


For real $\tau, Q_{n}(z, \tau)$ are called quasi-orthogonal L-polynomials. In particular $Q_{n}(z, 0)=Q_{n}(z)$. If $n$ is singular, then $Q_{n}(z, \tau)=c_{\tau} Q_{n}(z)$.

Let $n$ be non-singular and $\tau$ real. All the zeros of $Q_{n}(z, \tau)$ are real and simple, and except for a single value of $\tau$ the number of zeros is $n$. Let $t_{j}^{(n)}(\tau)$, $j=1,2, \ldots, n$, be these zeros. There exist positive constants $\lambda_{j}^{(n)}(\tau), j=1,2, \ldots, n$, such that the Gaussian quadrature formula

$$
\gamma(F)=\sum_{j=1}^{n} \lambda_{j}^{(n)}(\tau) F\left(t_{j}^{(n)}(\tau)\right)
$$

is valid for all $f \in \mathscr{R}_{-2 m, 2 m-2}$ when $n=2 m$ and for all $F \in \mathscr{R}_{-2 m, 2 m}$ when $n=2 m+1$ (see Theorems 6.2 and 6.3 of [4]).

For more detailed information on the concepts discussed in this section, see [4], [7] and [13].

\section{Associated Laurent polynomials}

Let $L(t, z)$ be an $L$-polynomial in $t$, i.e. $L(t, z)=\sum_{j=p}^{q} a_{j}(z) t^{j}$. To emphasize that $\gamma$ is operating on $t$ in $L(t, z)$ we write

$$
\gamma(L(t, z))=\gamma_{t}(L(t, z))=\sum_{j=p}^{q} a_{j}(z)(-1)^{j} c_{j} .
$$

If $\psi$ is any solution of the SHMP associated with the sequence $C$, then clearly $\gamma_{t}(L(t, z))=\int_{-\infty}^{\infty} L(t, z) d \psi(t)$.

For every $z \in \mathbf{C}$ let $D_{z}$ denote the difference quotient operator on $\mathscr{R}$ defined by $\left(D_{z} P\right)(t)=(P(t)-P(z)) /(t-z)$. We note that $D_{z} P \in \mathscr{R}_{p, q-1}$ when $P \in \mathscr{R}_{p, q}$.

For every quasi-orthogonal $L$-polynomial $Q_{n}(z, \tau)$ we define $P_{n}(z, \tau), n \geqslant 1$, by

$$
P_{n}(z, \tau)=\gamma_{t}\left(D_{z} Q_{n}(t, \tau)\right)=\gamma_{t}\left[\frac{Q_{n}(t, \tau)-Q_{n}(z, \tau)}{t-z}\right]
$$

We write $P_{n}(z)$ for $P_{n}(z, 0)$. We define $P_{0}(z) \equiv 0$. The $L$-polynomial $A_{n}(z, \tau)=$ $z P_{n}(z, \tau)$ belongs to $\mathscr{R}_{n}$. In analogy with the situation in the theory of ordinary orthogonal polynomials, $A_{n}(z, \tau)$ may be called the L-polynomial associated with $Q_{n}(z, \tau)$. We shall here find it convenient to work mostly with $P_{n}(z, \tau)$.

Next we formally state a simple lemma, some special cases of which will be repeatedly used in the sequel.

LEMMA 2.1. The formula $\gamma_{t}\left(D_{z}\left(P Q_{n}\right)\right)=P(z) P_{n}(z)$ is valid for every $P \in \mathscr{R}_{-m, m}$ when $n=2 m$, and for every $P \in \mathscr{R}_{-m, m+1}$ when $n=2 m+1$. 
Proof. We may write

$$
\gamma_{t}\left[D_{z}\left(P Q_{n}\right)\right]=P(z) \gamma_{t}\left(D_{z} Q_{n}\right)+\gamma_{t}\left[Q_{n} D_{z} P\right]
$$

which implies that the desired formula is valid whenever $D_{z} P \in \mathscr{R}_{n-1}$.

From Lemma 2.1 it immediately follows that we may write

$$
P_{2 m}(z, \tau)=P_{2 m}(z)-\tau z P_{2 m-1}(z), \quad P_{2 m+1}(z, \tau)=P_{2 m+1}(z)-\tau z^{-1} P_{2 m}(z) .
$$

We shall now prove some important identities involving the functions $Q_{n}(z)$ and $P_{n}(z)$. These identities are analogous to the Christoffel-Darboux formulas and related formulas, which occur in the theory of ordinary orthogonal polynomials. Note, however, that in our case 3-term recursion formulas for the functions $Q_{n}(z)$ and $P_{n}(z)$ are not available. For the classical results see, for example, [2, Chapter 2].

LEMMA 2.2. The following equalities hold for an arbitrary complex number $z \neq 0$

$$
\begin{aligned}
& z Q_{2 m}(z) P_{2 m-1}(z)-z Q_{2 m-1}(z) P_{2 m}(z)=\frac{q_{2 m,-m}}{q_{2 m-1,-m}} \\
& z Q_{2 m}(z) P_{2 m+1}(z)-z Q_{2 m+1}(z) P_{2 m}(z)=\frac{q_{2 m+1, m}}{q_{2 m, m}}
\end{aligned}
$$

ProOF. We can write $Q_{n}(z) P_{n-1}(z)-Q_{n-1}(z) P_{n}(z)=\gamma_{t}\left[Q_{n} \cdot\left(D_{z} Q_{n-1}\right)\right]-$ $\gamma_{t}\left[Q_{n-1} \cdot\left(D_{z} Q_{n}\right)\right]$, and this expression equals $-\gamma_{t}\left[Q_{n-1} \cdot\left(D_{z} Q_{n}\right)\right]$, since $Q_{n}$ is orthogonal to $D_{z} Q_{n-1}$. By using the expressions for $\gamma\left(t^{-m} Q_{2 m-1}(t)\right)$ and $\gamma\left(t^{m} Q_{2 m}(t)\right)$ from Section 1 we easily obtain $\gamma_{t}\left[Q_{2 m-1} \cdot\left(D_{z} Q_{2 m}\right)\right]=$ $-\left(q_{2 m,-m} / q_{2 m-1,-m}\right) z^{-1}$ and $\gamma_{t}\left[Q_{2 m} \cdot\left(D_{z} Q_{2 m+1}\right)\right]=\left(q_{2 m+1,-(m+1)} / q_{2 m, m}\right) z^{-1}$, from which formulas (A) and (B) follow.

Proposition 2.3. The following equalities hold for arbitrary complex numbers $z, \zeta \neq 0$

(A)

$$
z Q_{2 m-1}(z) Q_{2 m}(\zeta)-\zeta Q_{2 m}(z) Q_{2 m-1}(\zeta)=\frac{q_{2 m,-m}}{q_{2 m-1,-m}}(z-\zeta) \sum_{j=0}^{2 m-1} Q_{j}(z) Q_{j}(\zeta) ;
$$

(B)

$$
z Q_{2 m+1}(z) Q_{2 m}(\zeta)-\zeta Q_{2 m}(z) Q_{2 m+1}(\zeta)=\frac{q_{2 m+1, m}}{q_{2 m, m}}(z-\zeta) \sum_{j=0}^{2 m} Q_{j}(z) Q_{j}(\zeta)
$$


ProOF. We sketch a proof of (A); the proof of (B) is similar. We note that the function $F(\zeta)=(\zeta-z)^{-1}\left[z Q_{2 m-1}(z) Q_{2 m}(\zeta)-\zeta Q_{2 m}(z) Q_{2 m-1}(\zeta)\right]$ belongs to $\mathscr{R}_{2 m-1}$, and so we may write $F(\zeta)=\sum_{j=0}^{2 m-1}\left\langle F, Q_{j}\right\rangle Q_{j}(\zeta)$. Taking into account Lemma 2.1 and the fact that $\left(D_{z} Q_{j}\right)(\zeta) \in \mathscr{R}_{2 m-1}$ and $\zeta \cdot\left(D_{z} Q_{j}\right)(\zeta) \in \mathscr{R}_{2 m-1}$ for $j \leqslant 2 m-1$, we get by simple calculation that $\left\langle F, Q_{j}\right\rangle=$ $Q_{j}(z)\left[z Q_{2 m-1}(z) P_{2 m}(z)-z Q_{2 m}(z) P_{2 m-1}(z)\right]$. Substitution from Lemma 2.2 for the expression in the last bracket leads to the desired formula.

Proposition 2.4. The following equalities hold for arbitrary complex numbers $z, \xi \neq 0$

(A)

$$
\begin{aligned}
& z P_{2 m-1}(z) Q_{2 m}(\zeta)-\zeta P_{2 m}(z) Q_{2 m-1}(\zeta) \\
& =\frac{q_{2 m,-m}}{q_{2 m-1,-m}}\left[1+(z-\zeta) \sum_{j=1}^{2 m-1} P_{j}(z) Q_{j}(\zeta)\right] .
\end{aligned}
$$

(B) $z P_{2 m+1}(z) Q_{2 m}(\zeta)-\zeta P_{2 m}(z) Q_{2 m+1}(\zeta)$

$$
=\frac{q_{2 m+1, m}}{q_{2 m, m}}\left[1+(z-\zeta) \sum_{j=1}^{2 m} P_{j}(z) Q_{j}(\zeta)\right] .
$$

Proof. The formulas are obtained by using the operator $D_{z}$ and then the functional $\gamma_{t}$ on both sides of the equalities in Proposition 2.3(A) and Proposition 2.3(B) (taking into account Lemma 2.1).

Proposition 2.5. The following equalities hold for arbitrary complex numbers $z, \zeta \neq 0$

(A)

$$
\begin{aligned}
& z P_{2 m-1}(z) P_{2 m}(\zeta)-\zeta P_{2 m}(z) P_{2 m-1}(\zeta)=\frac{q_{2 m,-m}}{q_{2 m-1,-m}}(z-\zeta) \sum_{j=1}^{2 m-1} P_{j}(z) P_{j}(\zeta) \\
& \text { (B) } \quad z P_{2 m+1}(z) P_{2 m}(\zeta)-\zeta P_{2 m}(z) P_{2 m+1}(\zeta)=\frac{q_{2 m+1, m}}{q_{2 m, m}}(z-\zeta) \sum_{j=1}^{2 m} P_{j}(z) P_{j}(\zeta)
\end{aligned}
$$

Proof. The result can be obtained from Proposition 2.4 in the same way as Proposition 2.4 is obtained from Proposition 2.3.

In the following, $T_{n}(z, a, b)$ shall denote the expression $a P_{n}(z)+b Q_{n}(z)$, where $z, a$ and $b$ are arbitrary complex numbers, $z \neq 0$. 
THEOREM 2.6. The following equalities hold for arbitrary complex numbers $z, \zeta, a, b, \alpha, \beta(z, \zeta \neq 0)$ :

(A)

$$
\begin{aligned}
& z T_{2 m-1}(z, a, b) T_{2 m}(\zeta, \alpha, \beta)-\zeta T_{2 m}(z, a, b) T_{2 m-1}(\zeta, \alpha, \beta) \\
& \quad=\frac{q_{2 m,-m}}{q_{2 m-1,-m}}\left[(a \beta-\alpha b)+(z-\zeta) \cdot \sum_{j=0}^{2 m-1} T_{j}(z, a, b) T_{j}(\zeta, \alpha, \beta)\right] \\
& z T_{2 m+1}(z, a, b) T_{2 m}(\zeta, \alpha, \beta)-\zeta T_{2 m}(z, a, b) T_{2 m+1}(\zeta, \alpha, \beta) \\
& \quad=\frac{q_{2 m+1, m}}{q_{2 m, m}}\left[(a \beta-\alpha b)+(z-\zeta) \cdot \sum_{j=0}^{2 m} T_{j}(z, a, b) T_{j}(\zeta, \alpha, \beta)\right]
\end{aligned}
$$

Proof. By multiplying out the terms on the left side in (A) and (B) and using the equalities of Proposition 2.3, Proposition 2.4 and Proposition 2.5, we obtain the desired formulas.

In the following, $w$ shall denote an arbitrary complex number different from zero. An asterisk denotes complex conjugation.

COROLlary 2.7. The following equalities hold when $\operatorname{Im} z \neq 0$ :

$$
\begin{gathered}
z T^{2 m-1}(z, 1, w) T_{2 m}(z, 1, w)^{*}-z^{*} T_{2 m-1}(z, 1, w)^{*} T_{2 m}(z, 1, w) \\
=(z-z)^{*} \frac{q_{2 m,-m}}{q_{2 m-1, m}}\left[-\frac{w-w^{*}}{z-z^{*}}+\sum_{j=0}^{2 m-1}\left|T_{j}(z, 1, w)\right|^{2}\right] ; \\
z T_{2 m+1}(z, 1, w) T_{2 m}(z, 1, w)^{*}-z^{*} T_{2 m+1}(z, 1, w)^{*} T_{2 m}(z, 1, w) \\
=(z-z)^{*} \frac{q_{2 m+1, m}}{q_{2 m, m}}\left[-\frac{w-w^{*}}{z-z^{*}}+\sum_{j=0}^{2 m}\left|T_{j}(z, 1, w)\right|^{2}\right] .
\end{gathered}
$$

PRoof. Note that $Q_{n}(z)$ and $P_{n}(z)$ are $L$-polynomials with real coefficients. Therefore $Q_{n}(z)^{*}=Q_{n}\left(z^{*}\right), P_{n}(z)^{*}=P_{n}\left(z^{*}\right)$. By setting $\zeta=z^{*}, a=\alpha=1$, $b=w, \beta=w^{*}$ in Theorem 2.6, we obtain the desired formula.

\section{Nested circles}

For arbitrary complex numbers $\tau$ and non-real numbers $z$ we define $w_{n}(z, \tau)$ by $w_{n}(z, \tau)=-P_{n}(z, \tau) / Q_{n}(z, \tau)$. Thus

$$
\begin{gathered}
w_{2 m}(z, \tau)=-\frac{P_{2 m}(z)-\tau z P_{2 m-1}(z)}{Q_{2 m}(z)-\tau z Q_{2 m-1}(z)}, \\
w_{2 m+1}(z, \tau)=-\frac{P_{2 m+1}(z)-\tau z^{-1} P_{2 m}(z)}{Q_{2 m+1}(z)-\tau z^{-1} Q_{2 m}(z)} .
\end{gathered}
$$


For every non-singular index $n$ the point $w_{n}(z, \tau)$ describes a circle $\Gamma_{n}(z)$ in the complex plane when $\tau$ varies over the extended real axis (the point $\infty$ included). (Recall that $Q_{n}(z, \tau) \neq 0$ when $\tau$ is real and $z$ non-real; cf. Section 1.) Let $\Delta_{n}(z)$ denote the closed disc bounded by $\Gamma_{n}(z)$. We shall show that these discs have important properties in common with the nested discs encountered in the classical case.

THEOREM 3.1. For a non-singular index $n$ the disc $\Delta_{n}(z)$ is given by the inequality

$$
\sum_{j=0}^{n-1}\left|T_{j}(z, 1, w)\right|^{2} \leqslant \frac{w-w^{*}}{z-z^{*}} .
$$

Proof. Assume for example that $n=2 m$. Since $2 n$ is non-singular we have $q_{2 m,-m} \neq 0$, and it follows from Corollary 2.7 that $w \in \Gamma_{2 m}(z)$ if and only if

$$
\sum_{j=0}^{2 m-1}\left|T_{j}(z, 1, w)\right|^{2}-\frac{w-w^{*}}{z-z^{*}}=0 .
$$

A standard mapping argument shows that

$$
\sum_{j=0}^{2 m-1}\left|T_{j}(z, 1, w)\right|^{2}-\frac{w-w^{*}}{z-z^{*}}<0
$$

when $z$ is inside $\Gamma_{2 m}(z)$. The argument for $n=2 m+1$ is similar.

THEOREM 3.2. Let $m$ and $n$ be non-singular indices, $m>n$. Then $\Delta_{m}(z) \subset \Delta_{n}(z)$.

Proof. This is immediate from Theorem 3.1.

THEOREM 3.3. For a non-singular index $n$ the radius $\rho_{n}(z)$ of the disc $\Delta_{n}(z)$ is given by

$$
\rho_{n}(z)=\left[\left|z-z^{*}\right| \cdot \sum_{j=0}^{n-1}\left|Q_{j}(z)\right|^{2}\right]^{-1}
$$

Proof. Assume for example that $n=2 m$. A standard result on Möbius transformations show that the radius of $\Delta_{2 m}(z)$ equals the modulus of the expression

$$
\begin{aligned}
& {\left[z P_{2 m}(z) Q_{2 m-1}(z)-z P_{2 m-1}(z) Q_{2 m}(z)\right] } \\
& \cdot\left[z^{*} Q_{2 m}(z) Q_{2 m-1}\left(z^{*}\right)-z Q_{2 m-1}(z) Q_{2 m}\left(z^{*}\right)\right]^{-1} .
\end{aligned}
$$

Substituting from Lemma 2.2 and Proposition 2.3 (with $\zeta=z^{*}$ ) we get $\rho_{2 m}(z)=$ $\left[\left|z-z^{*}\right| \sum_{j=0}^{n-1}\left|Q_{j}(z)\right|^{2}\right]^{-1}$. Similarly with $n=2 m+1$, we obtain the desired formula for $\rho_{2 m+1}(z)$. 
If the index $2 m$ is singular, then $q_{2 m,-m}=0$, and the arguments leading to the equation for the circle $\Gamma_{2 m}(z)$ and the radius $\rho_{2 m}(z)$ fail. From the formula $Q_{2 m}(z)=\left(q_{2 m,-(m-1)} / q_{2 m-1,-m}\right) z Q_{2 m-1}(z)$ (see Section 1) and Lemma 2.1 it follows that $P_{2 m}(z)=\left(q_{2 m,-(m-1)} / q_{2 m-1,-m}\right) z P_{2 m-1}(z)$, and so

$$
w_{2 n}(z, \tau)=-\frac{A z P_{2 m-1}(z)-\tau z P_{2 m-1}(z)}{A z Q_{2 m-1}(z)-\tau z Q_{2 m-1}(z)}=-\frac{P_{2 m-1}(z)}{Q_{2 m-1}(z)} .
$$

Thus $\Gamma_{2 m}(z)$ degenerates to a point in this case.

Similarly, if the index $2 m+1$ is singular, then $\Gamma_{2 m+1}(z)$ degenerates to the point $P_{2 m}(z) / Q_{2 m}(z)$.

We define the set $\Delta_{\infty}(z)$ to be the intersection of all the dises $\Delta_{n}(z)$ for non-singular indices $n$. Since $\Delta_{m}(z) \subset \Delta_{n}(z)$ for $m>n$ (Theorem 3.2), the set $\Delta_{\infty}(z)$ is a point or a closed disc, the radius $\rho(z)$ being given by

$$
\rho(z)=\lim _{\substack{n \rightarrow \infty \\ n \text { non-singular }}} \rho_{n}(z) .
$$

THEOREM 3.4. If $\Delta_{\infty}(z)$ is a disc, then $\sum_{j=0}^{\infty}\left|Q_{j}(z)\right|^{2}<\infty$ and $\sum_{j=1}^{\infty}\left|P_{j}(z)\right|^{2}<\infty$. If $\Delta_{\infty}(z)$ is a point, then $\sum_{j=0}^{\infty}\left|Q_{j}(z)\right|^{2}=\infty$ and $\sum_{j=1}^{\infty}\left|P_{j}(z)\right|^{2}=\infty$.

Proof. It follows from Theorem 3.3 that $\sum_{j=0}^{\infty}\left|Q_{j}(z)\right|^{2}<\infty$ if $\Delta_{\infty}(z)$ is a disc and $\sum_{j=0}^{\infty}\left|Q_{j}(z)\right|^{2}=\infty$ if $\Delta_{\infty}(z)$ is a point. Let $w$ be a point in $\Delta_{\infty}(z)$. Then $\sum_{j=0}^{\infty}\left|P_{j}(z)+w Q_{j}(z)\right|^{2}<\infty$ (Theorem 3.1), and it follows that $\sum_{j=1}^{\infty}\left|P_{j}(z)\right|^{2}=\infty$ if and only if $\sum_{j=0}^{\infty}\left|Q_{j}(z)\right|^{2}=\infty$ (note that $w \neq 0$, for example by Theorem 3.1).

THEOREM 3.5 (Theorem of invariability). If $\Delta_{\infty}(\zeta)$ is a disc for some non-real number $\zeta$, then $\Delta_{\infty}(z)$ is a disc for very non-real number $z$.

Proof. Assume that $\Delta_{\infty}(\zeta)$ is a disc. It follows from Theorem 3.4 by elementary estimates by

$$
\sum_{n=0}^{\infty} \sum_{j=0}^{n-1}\left|Q_{j}(\zeta) P_{n}(\zeta)-Q_{n}(\zeta) P_{j}(\zeta)\right|^{2}<\infty
$$

Let $z \neq \zeta, \operatorname{Im} z \neq 0$, and set $M=\max \left\{|z-\zeta|,\left|\zeta z^{-1}(z-\zeta)\right|\right\}$. Let $0<\varepsilon<1$. By Theorem 3.5 and the foregoing remark there exists a natural number $\nu$ such that

$$
\sum_{n=\nu}^{\infty} \sum_{j=1}^{n-1}\left|Q_{j}(\zeta) P_{n}(\zeta)-Q_{n}(\zeta) P_{j}(\zeta)\right|^{2}<\frac{\varepsilon^{2}}{M^{2}}
$$

and

$$
\sum_{n=\nu}^{\infty}\left|Q_{n}(\zeta)\right|^{2}<\varepsilon^{2}
$$


(cf. [1, p. 17]). Straightforward calculations show that the formulas

$$
Q_{2 m}(z)=Q_{2 m}(\zeta)+(z-\zeta) \sum_{j=0}^{2 m-1} Q_{j}(z)\left[Q_{j}(\zeta) P_{2 m}(\zeta)-Q_{2 m}(\zeta) P_{j}(\zeta)\right]
$$

and

$$
\begin{aligned}
Q_{2 m+1}(z)= & Q_{2 m+1}(\zeta) \\
& +z^{-1} \zeta(z-\zeta) \sum_{j=0}^{2 m} Q_{j}(z)\left[Q_{j}(\zeta) P_{2 m+1}(\zeta)-Q_{2 m+1}(\zeta) P_{j}(\zeta)\right]
\end{aligned}
$$

hold. By using these relations, the Schwartz inequality and Minkowski's inequality, we obtain for every $N \geqslant \nu$ the estimate $\sum_{n=\nu}^{N}\left|Q_{n}(z)\right|^{2} \leqslant \varepsilon\left[1+\sum_{n=0}^{\nu}\left|Q_{n}(z)\right|^{2}\right]$. This shows that $\sum_{n=\nu}^{\infty}\left|Q_{n}(z)\right|^{2}<\infty$, and the result follows from Theorem 3.4.

In view of Theorem 3.5 we may use the terms limit circle case and limit point case without reference to any particular point $z$.

\section{Stieltjes transforms}

Let $\psi$ be a bounded, real-valued, non-decreasing function. The Stieltjes transform $\hat{\psi}$ of $\psi$ is given by

$$
\hat{\psi}(z)=\int_{-\infty}^{\infty} \frac{d \psi(t)}{t-z}, \quad \operatorname{Im} z \neq 0 .
$$

The function $\psi$ is said to represent the functional $\gamma$ (introduced in Section 1) on a subspace $\mathscr{H}$ of $\mathscr{R}$ if $\gamma(f)=\int_{-\infty}^{\infty} f(t) d \psi(t)$ for every $f \in \mathscr{H}$. In particular the distribution function $\psi$ represents $\gamma$ on $\mathscr{R}$ if and only if it is a solution of the SHMP for the sequence $C$. (It is easily proved that when $(\mathrm{H})$ is satisfied, then every bounded real-valued non-decreasing function representing $\gamma$ on $\mathscr{R}$ has infinitely many points of increase; see Proposition 2.2 of [4].)

We define the subsets $\Sigma_{p, q}(z)$ of the complex plane as follows:

$$
\sum_{p, q}(z)=\left\{\hat{\psi}(z): \psi \text { is a representing function for } \gamma \text { on } \mathscr{R}_{p, q}\right\} \text {. }
$$

THEOREM 4.1. $\Sigma_{p, q}(z)$ is a compact, convex set.

Proof. Compactness follows by a simple application of Helly's theorems. Convexity follows from the fact that if $\psi_{1}$ and $\psi_{2}$ are representing functions for $\gamma$ on $\mathscr{R}_{p, q}$, then $\psi=\theta \psi_{1}+(1-\theta) \psi_{2}, 0<\theta<1$, is also a representing function for $\gamma$ on $\mathscr{R}_{p, q}$. 
We recall the Gaussian quadrature formulas associated with the quasi-orthogonal $L$-polynomials $Q_{n}(z, \tau)$ as described in Section 1 . For non-singular $n$, real $t$ and $\tau$ we define the function $\psi_{n}(t, \tau)$ by

$$
\psi_{j}(t, \tau)=\sum\left\{\lambda_{j}^{(n)}(\tau): t_{j}^{(n)}(\tau) \leqslant t\right\} .
$$

This function is bounded and non-decreasing in $t$.

It follows immdiately from the quadrature formulas that except for a single value of $\tau, \psi_{n}(t, \tau)$ represents $\gamma$ on $\mathscr{R}_{-2 m, 2 m-2}$ when $n=2 m$, and on $\mathscr{R}_{-2 m, 2 m}$ when $n=2 m+1$. Thus $\hat{\psi}_{2 m}(z, \tau) \in \sum_{-2 m, 2 m-2}(z), \hat{\psi}_{2 m+1}(z, \tau) \in \sum_{-2 m, 2 m}(z)$.

In terms of $w_{n}(z, \tau)$ which was defined in Section 3, we have the following result.

THEOREM 4.2. For every non-singular $n$ and $\operatorname{Im} z \neq 0$ we have $\hat{\psi}_{n}(z, \tau)=w_{n}(z, \tau)$.

Proof. From the quadrature formulas we get for $\tau$ fixed (taking into account that $\left.Q_{n}\left(t_{j}^{(n)}(\tau), \tau\right)=0\right)$ :

$$
\begin{aligned}
P_{n}(z, \tau) & =\gamma_{t}\left(D_{z} Q_{n}(t, \tau)\right)=-\sum_{j=1}^{n} \lambda_{j}^{(n)}(\tau) \frac{Q_{n}(z, \tau)}{\left[t_{j}^{(n)}(\tau)-z\right]} \\
& =-Q_{n}(z, \tau) \int_{-\infty}^{\infty} \frac{d \psi_{n}(t, \tau)}{t-z}=-Q_{n}(z, \tau) \hat{\psi}_{n}(z, \tau)
\end{aligned}
$$

THEOREM 4.3. The following equalities hold:

$$
\begin{aligned}
& \Delta_{2 m}(z)=\sum_{-2 m, 2 m-2}(z) \text { when } 2 m \text { is non-singular; } \\
& \Delta_{2 m+1}=\sum_{-2 m, 2 m}(z) \text { when } 2 m+1 \text { is non-singular. }
\end{aligned}
$$

Proof. It follows from Theorem 4.2 and the remarks preceding it that, except for possibly two points, $\Gamma_{2 m}(z) \subset \sum_{-2 m, 2 m-2}(z)$ if $n=2 m, \Gamma_{2 m+1}(z) \subset$ $\sum_{-2 m, 2 m}(z)$ if $n=2 m+1$. (The two exceptional points correspond to $\tau=\infty$ and the value of $\tau$ for which the Gaussian quadrature formula may fail.) Hence by Theorem 4.1 we get $\Delta_{2 m}(z) \subset \sum_{-2 m, 2 m-2}(z), \Delta_{2 m+1}(z) \subset \sum_{-2 m, 2 m}(z)$.

On the other hand let $\psi$ be a function which represents $\gamma$ on $\mathscr{R}_{-2 m, 2 m-1}$ (respectively $\mathscr{R}_{-2 m, 2 m}$ ). Then the bilinear forms defined by $\gamma$ and by $\psi$ coincide on $\mathscr{R}_{2 m-1}$ (respectively $\mathscr{R}_{2 m}$ ). Thus the system $\left\{Q_{0}, Q_{1}, \ldots, Q_{n-1}\right\}$ is orthonormal with respect to the function $\psi$ both in case (A) $(n=2 m)$ and in case (B) $(n=2 m+1)$. Set $w=\hat{\psi}(z)$. Using Bessel's inequality for the function $f(t)=$ $1 /(t-z)$, observing that $\int_{-\infty}^{\infty} d \psi(t) /|t-z|^{2}=\left(w-w^{*}\right) /\left(z-z^{*}\right)$ and $\left\langle f, Q_{j}\right\rangle$ $=P_{j}(z)+w Q_{j}(z)$, we get $\sum_{j=0}^{n-1}\left|P_{j}(z)+w Q_{j}(z)\right|^{2} \leqslant\left(w-w^{*}\right) /\left(z-z^{*}\right)$. It follows from Theorem 3.1 that $\hat{\psi}(z)=w \in \Delta_{n}(z)$. 


\section{The uniqueness theorem}

We define the subset $\Sigma(z)$ of the complex plane as follows: $\Sigma(z)=\{\hat{\psi}(z): \psi$ is a representing distribution function for $\psi$ on $\mathscr{R}\}$. We recall that two successive orthogonal $L$-polynomials $Q_{n}(z)$ and $Q_{n+1}(z)$ are not both singular; see Section 1 (cf. Corollary 3.4 of [4]). It follows from Theorem 4.3 that for every $m$, either $\Delta_{2 m}(z)=\Sigma_{-2 m, 2 m-2}(z)$ or $\Delta_{2 m+1}(z)=\Sigma_{-2 m, 2 m}(z)$.

THEOREM 5.1. For every non-real $z$ we have $\Sigma(z)=\Delta_{\infty}(z)$.

Proof. The inclusion $\Sigma(z) \subset \Delta_{\infty}(z)$ follows immediately from Theorem 4.3 and the fact that a representing distribution function for $\gamma$ on $\mathscr{R}$ also is a representing function on every subspace $\mathscr{R}_{p, q}$.

Again by Theorem 4.3 we get $\Delta_{\infty}(z) \subset \sum_{-2 m, 2 m}(z)$ for every $m$. Thus for every $w \in \Delta_{\infty}(z)$ and every $m$ there exists a function $\psi_{m}$ representing $\gamma$ on $\mathscr{R}_{-2 m, 2 m}$ such that $\hat{\psi}_{n}(z)=w$. The sequence $\left\{\psi_{m}\right\}$ is uniformly bounded since $\int_{-\infty}^{\infty} d \psi_{m}(t)$ $=c_{0}$. A standard argument involving Helly's selection theorems establishes the existence of a distribution function $\psi$ which represents $\gamma$ on $\mathscr{R}$ such that $\hat{\psi}(z)=w$ (cf. Section 7 of [4]), and hence $\Delta_{\infty}(z) \subset \Sigma(z)$.

THEOREM 5.2. The SHMP has an essentially unique solution in the limit point case, infinitely many solutions in the limit circle case.

Proof. This follows immediately from Theorem 5.1 and the essential uniqueness of the inverse Stieltjes transform.

\section{References}

[1] N. I. Akiezer, The classical moment problem and some related questions in analysis (Hafner Publishing Company, New York, 1965).

[2] C. Brezinski, Padé-type approximation and general orthogonal polynomials (Birkhäuser Verlag, Basel, Boston, Stuttgart, 1980).

[3] Z. Cobindarajula, 'Recurrence relations for the inverse moments of the positive binomial variable', J. Amer. Statist. Assoc. 57 (1963), 468-473.

[4] William B. Jones, Olav Njàstad and W. J. Thron, 'Orthogonal Laurent polynomials and the strong Hamburger Moment Problem', J. Math. Anal. Appl. 98 (1984), 528-554.

[5] William B. Jones, Olav Njåstad and W. J. Thron, 'Continued fractions and strong Hamburger moment problems', Proc. London Math. Soc. (3), 47 (1983), 363-384.

[6] William B. Jones, Olav Njåstad and W. J. Thron, 'Two-point Padé expansions for a family of analytic functions', J. Comput. Appl. Math. 9 (1983), 105-123. 
[7] William B. Jones and W. J. Thron, 'Orthogonal Laurent polynomials and Gaussian quadrature', pp. 449-455, Quantum mechanics in mathematics, chemistry and physics, (Eds., K. Gustafson and W. P. Reinhardt), Plenum Publishing Corp., New York, (1981).

[8] William B. Jones and W. J. Thron, 'Survey of continued fractions methods of solving moment problems and related topics', pp. 4-37, Analytic theory of continued fractions, Proceedings, Loen, Norway, 1981, Lecture Notes in Mathematics, No. 932, Springer Verlag, Berlin (1982).

[9] William B. Jones, W. J. Thron and H. Waadeland, 'A strong Stieltjes moment problem', Trans. Amer. Math. Soc. 261 (1980), 503-528.

[10] D. G. Kabe, 'Inverse moments and discrete distributions', Canad. J. Statist. 4 (1976), 133-142.

[11] W. Mendenhall and E. H. Lehman, Jr., 'An approximation of the negative moments of the positive binomial useful in life testing', Technometrics 2 (1960), 227-242.

[12] H. J. Landau, 'The classical moment problem: Hilbertian proofs', J. Functional Analysis 38 (1980), 255-272.

[13] Olav Njăstad and W. J. Thron, 'The theory of sequences of orthogonal $L$-polynomials', Padé approximants and continued fractions, 54-91, (Eds., Waadeland, Haakon and Wallin, Hans), Det Kongelige Norske Videnskabers Selskab, Skrifter (1983), No. 1.

[14] Olav Njåstad and W. J. Thron, 'Completely convergent APT-fractions and strong Hamburger moment problems with a unique solution', Det Kongelige Norske Videnskabers Selskab, Skrifter, No. 2 (1984), 1-7.

[15] J. A. Shohat and J. D. Tamarkin, The problem of moments (Mathematical Surveys No. 1, Amer. Math. Soc., Providence, R. I., 1943).

[16] D. L. Thomas, 'Reciprocal moments of linear combinations of exponential variates', J. Amer. Statist. Assoc. 71 (1976), 506-512.

Department of Mathematics

University of Trondheim

N-7034 Trondheim-NTH

Norway
Department of Mathematics

Campus Box 426

University of Colorado

Boulder, Colorado 80309 